\title{
Arterial Performance Management System Lexicon
}
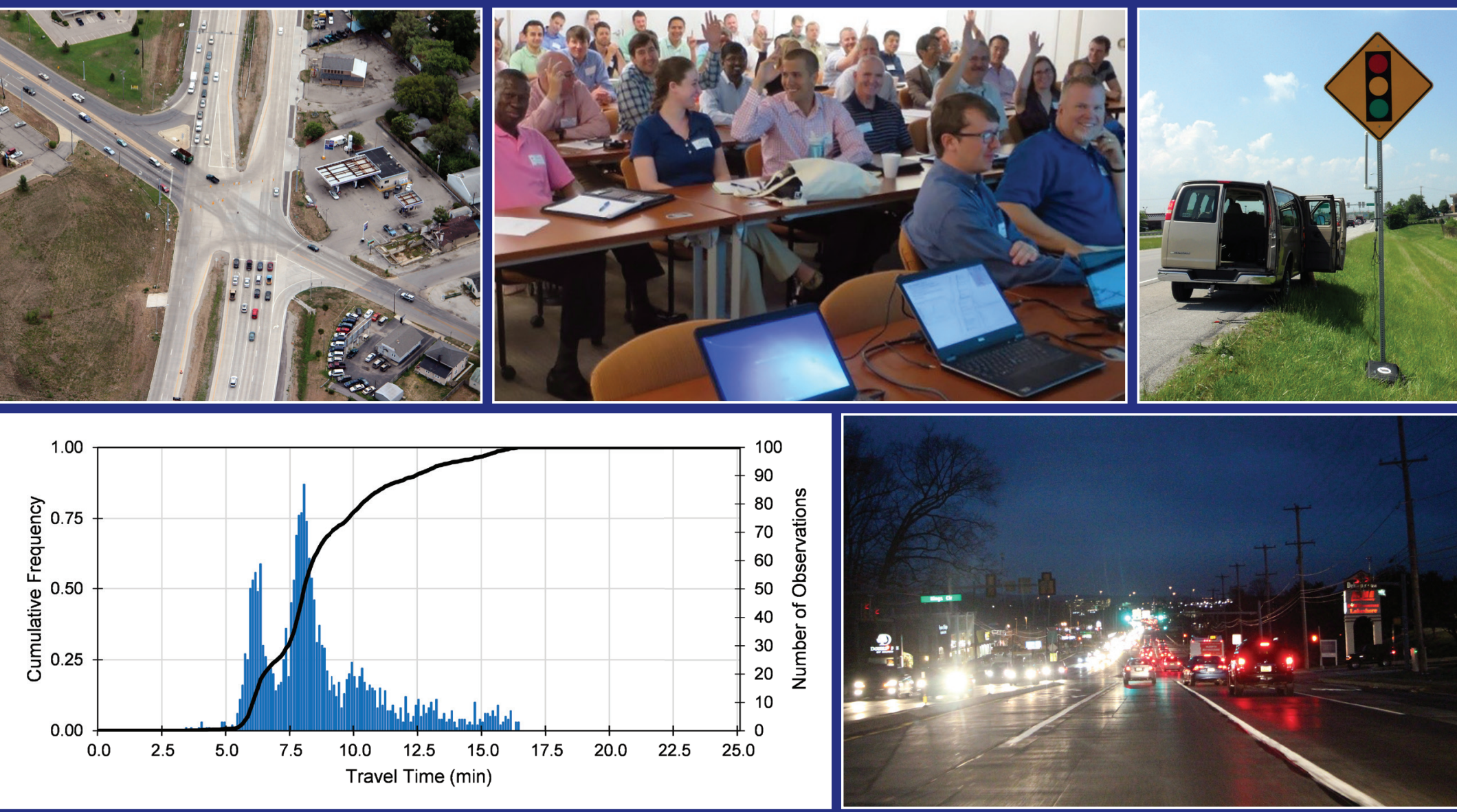

Stanley E. Young, Dennis So Ting Fong 


\section{Arterial Performance Management System Lexicon}

Stanley E. Young

Traffax, Inc.

Dennis So Ting Fong

Traffax, Inc.

SBIR Phase 3 Joint Transportation Research Project

Traffax, Inc.

Purdue University

May 4, 2015

\begin{tabular}{|l|l|}
\hline Deliverable Reference: & D2.1 Performance Measures Lexicon \\
\hline Contractor: & Traffax, Inc. \\
\hline Contract Number: & DTFH61-14-C-00035 \\
\hline Contract Term Start & $9 / 4 / 2014$ \\
\hline Contract Term End & 9/4/2017 \\
\hline Key Personnel & Stan Young, Darcy Bullock, Dennis So Ting Fong \\
\hline
\end{tabular}




\section{Recommended Citation}

Young, S. E., and D. S. T. Fong. Arterial Performance Management System Lexicon. Purdue University, West Lafayette, Indiana, 2017. https://doi.org/10.5703/1288284316565

\section{Acknowledgments}

This work was supported by Traffax/USDOT SBIR DTFH6114C00035. The contents of this paper reflect the views of the authors, who are responsible for the facts and the accuracy of the data presented herein, and do not necessarily reflect the official views or policies of the sponsoring organizations. These contents do not constitute a standard, specification, or regulation. 


\section{Table of Contents}

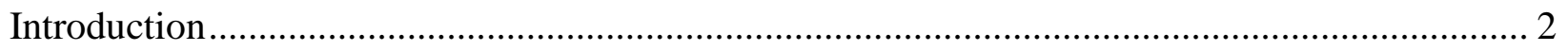

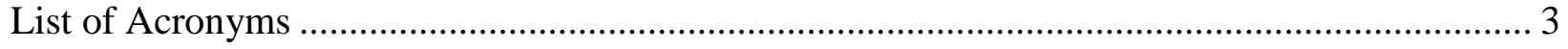

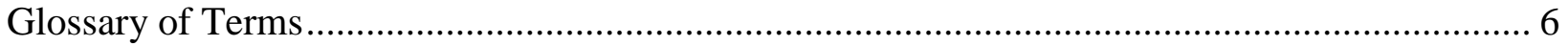

Arterial Performance Management Lexicon 1 


\section{Introduction}

The Lexicon for this project is intended to complement other project deliverables by providing additional detail and background. In order to facilitate the broadest possible audience the authors have incorporated a glossary of transportation terms. This glossary is the primary content of the Lexicon. Terms were included that have meaning specific to roadway transportation or traffic engineering.

In addition to a thorough glossary, other sections of the Lexicon contain additional material or ancillary details to the project deliverables or assist in implementation.

Lexicon sections include:

\section{List of Acronyms and Glossary of Terms}

Designed as a companion to the main project deliverables, the glossary provides a quick reference to the reader in order to provide background information, definitions, or clarification. The Lexicon for arterial performance measures, and specifically for this work, draws on concepts, language and ideas from the transportation engineering field in general, and at times these terms take on specific or narrower meanings than that used in common vernacular.

\section{Review of the state of practice for Outsourced Probe Data}

Outsourced probe data provides travel time and speed data for roadways without the need for deploying field equipment. It is based on the ability of vehicles (or their occupants) to self-report speed and location based on GPS instrumentation. At the time of this writing, the fidelity of outsourced field data has been well-documented for freeways, and its fidelity for signal controlled facilities is just beginning to be understood. Outsourced probe data, or frequently referred to simply as 'probe data', is discussed in conjunction with arterial monitoring, this section is included to provide an up-to-date assessment of probe data fidelity, and its issues with respect to using such data for signal controlled arterials. 


\section{List of Acronyms}

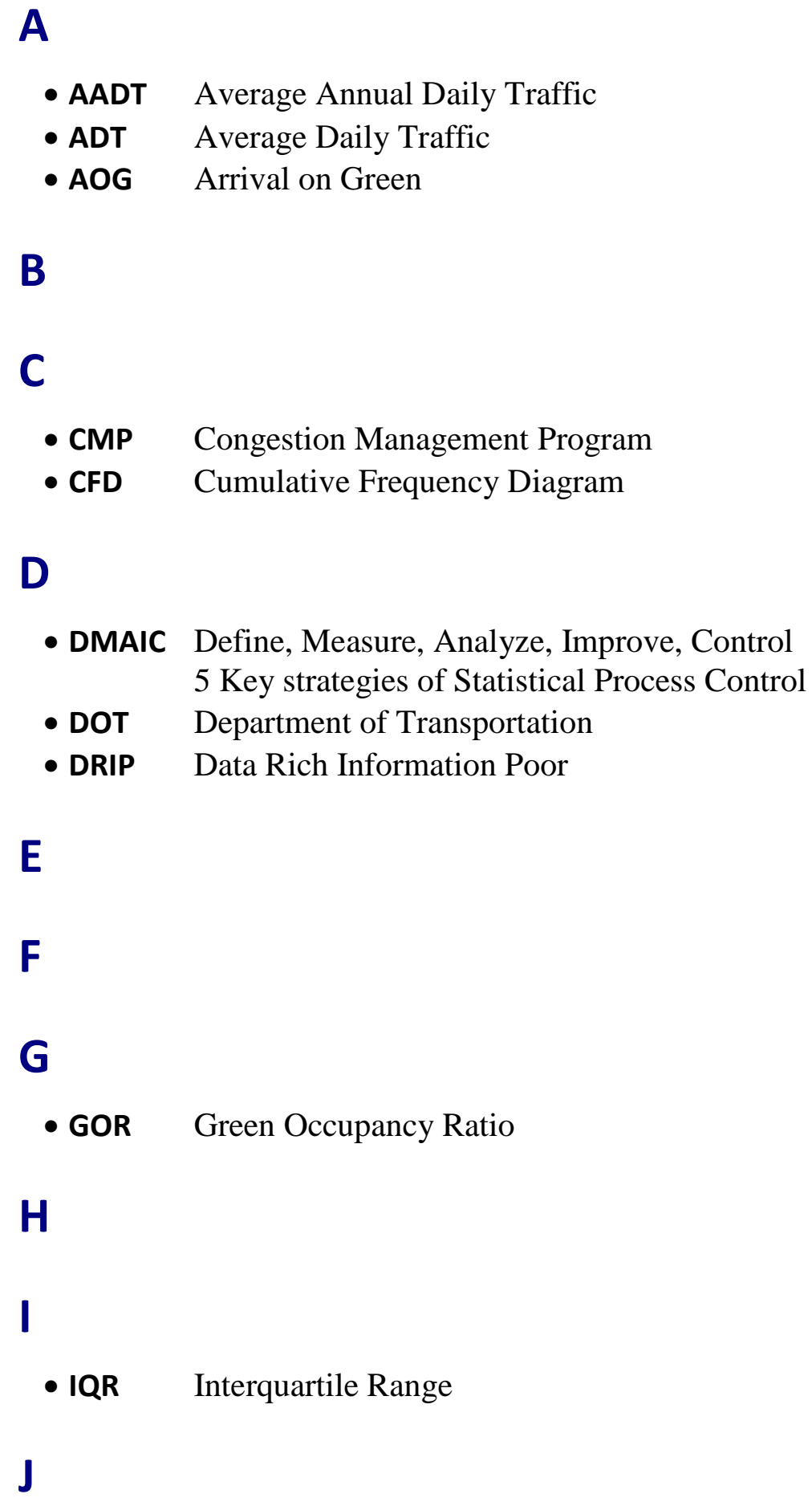

A

- AADT Average Annual Daily Traffic

- ADT Average Daily Traffic

- AOG Arrival on Green

B

C

- CMP Congestion Management Program

- CFD Cumulative Frequency Diagram

D

- DMAIC Define, Measure, Analyze, Improve, Control 5 Key strategies of Statistical Process Control

- DOT Department of Transportation

- DRIP Data Rich Information Poor

E

$\mathbf{F}$

G

- GOR Green Occupancy Ratio

H

I

- IQR Interquartile Range

J 
K

- KPI Key Performance Indicators

L

- LOS Level Of Service

M

- MAP-21 Moving Ahead for Progress in the $21^{\text {st }}$ Century Act

- MPO Metropolitan Planning Organization

$\mathbf{N}$

0

P

- POG Data Rich Information Poor

- PCD Purdue Coordination Diagram

Q

$\mathbf{R}$

- ROR Red Occupancy Ratio

S

- SPC Statistical Process Control

T

- TOD Time of Day

- TTI Texas Transportation Institute

- TTR Travel Time Reliability

U 


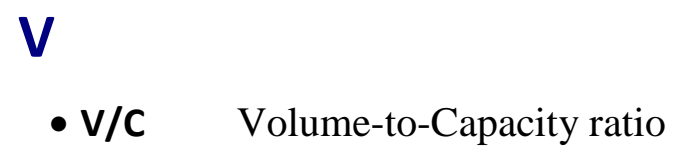

\section{W}




\section{Glossary of Terms}

A

Accessibility

With respect to roadway classification, accessibility refers to a road's ability to connect the traveler to goods and services through intersections and junctions with other roadways, business entrances, and driveways. Also known as 'land-access'.

\section{Actuation}

In signal control, this refers generally to the use of detection to modify signal behavior. Detectors are used to measure the arrival and/or presence of traffic. A signal controller may register "calls" for service based on detector information. Additionally, the controller might adjust the duration of green based on detector states, for example by applying a "passage" timer that holds the green for a certain amount of time after a specified detector becomes unoccupied.

\section{Agency-Oriented Objectives}

Term is used in contrast to 'user-oriented objectives' and reflects the issues and goals critical to the roadway owner or manager. Such objectives are typically identified from a system or funding perspective. Examples of such objectives may include: determining attainment of performance targets; ensuring funding investments provide a sufficient return; ensuring personnel and other resources are used optimally; influencing the traveling and / or route choice behavior of the public.

\section{Arterial or Arterial Highway}

Within the context of this report, an arterial is distinguished as a roadway having at-grade intersections. This report focuses on arterials with traffic signal control. Arterials provide both throughput and land-access. An arterial may connect to a freeway such that vehicles exiting the freeway can be distributed to the businesses and residences in a community via the arterial's connections with local roads.

\section{Arterial Management System}

The business process by which a network of arterial roadways are managed. This includes assessing performance, planning for improvements, determining funding requirements, and scheduling ongoing maintenance.

\section{Arterial Traffic Control System}

The electronic system that controls the display of traffic signals on a network of arterials. It includes the traffic signals, controllers, vehicle detectors and other sensors, and overall control software and algorithms used to safely and efficiently direct traffic.

Average Annual Daily Traffic (AADT)

The total traffic for a year divided by 365. 


\section{B}

\section{Box (Whisker) Plot}

A way of graphically depicting numerical data based on their quartiles. The limits of the box are based on the $25^{\text {th }}$ and $75^{\text {th }}$ percentiles. The mid-line of the plot is the $50^{\text {th }}$ percentile (or median). Optionally, lines extending vertically from the boxes (called whiskers) indicate the extent of the data and typically show the maximum and minimum values.

C

\section{Capacity}

The theoretical or anticipated maximum number of vehicles that can progress along a roadway or through an intersection. Capacity is calculated based on geometric attributes (such as number of lanes) and signal timing parameters (such as length of green time and cycle time). It is typically measured in vehicles per hour.

\section{Capacity Allocation}

Within a signalized intersection, this term refers to the allocation of green time to the competing traffic movements and their respective demand.

\section{Capacity Utilization}

A measure of the amount of provided capacity (that is, green time) that is being made use of by traffic. This can be measured by the volume-to-capacity ratio or by occupancy-based metrics.

\section{Capital Improvements}

Within the context of arterial management, capital improvements encompass physical changes to the roadway such as adding lanes, or adding turn bays. This is in contrast to noncapital improvements such as re-timing signals for increased efficiency.

\section{Congestion Management Program (CMP)}

Need definition

\section{Coordination (Signal Coordination)}

A traffic signal control strategy in which the green times of selected movements in a group of intersections are sequenced and scheduled to establish a reliable traffic flow pattern, usually intended to encourage the progression of traffic through multiple intersections.

\section{Corridor}

A logical length of roadway that may span multiple intersections, in contrast to a segment, which typically extends from one intersection to the next. A corridor is typically defined to comprise routes along which users would have an expectation of smooth traffic flow. A common example of a corridor is a numbered highway extending from a city center to a suburb, or between to cities. However, in practice, a corridor might include left or right turns, or take on different names as it passes through different jurisdictions.

\section{Cumulative Frequency Diagram (CFD)}

Also known as a cumulative distribution function, but specific to travel time along a corridor. Each point on a CFD reflects the percentage of traffic that progressed through the corridor at 
a travel time less or equal to ' $\mathrm{x}$ ' coordinate. A CFD is constructed from calculated percentiles of travel time. The graph always increases to the right. It provides a compact representation of the distribution of travel time along a corridor, and an efficient way to compare two or more time periods (such as before and after signal re-timing) of performance on a corridor.

\section{Cycle Failure}

In the context of the report, this refers to a condition where multiple split failures are occurring on different phases within a cycle, such that there is no longer any additional green time within the cycle that could be used to resolve the individual split failures.

\section{Cycle Length}

Within the context of traffic signal control, cycle length refers to the time period for the signal controller to service all of the competing movements present at an intersection. Generally, cycle length can be measured from the start of green time on a dominant movement, to the next start of green time for the same movement. Cycle lengths can range from 30 seconds to five minutes, but most are between 90 seconds and three minutes.

D

\section{Delay}

The travel time on a roadway in excess of the nominal travel time (sometimes referred to as free-flow travel time). For arterials, the primary source of delay is from traffic control devices such as traffic signals. Delay may be measured for individual movements, and for routes along a corridor or through a network.

\section{Demand, Traffic Demand}

Within the context of transportation, demand reflects the anticipated or measured number of vehicles traversing a roadway or through an intersection. It is typically expressed in vehicles per hour.

\section{Detector Occupancy (Occupancy or Occupancy Ratio)}

The percent of time that one or more vehicles are within the detection zone of a sensor. For example, an inductive loop sensor located prior to the stop bar of an intersection will detect the presence of a vehicle and the percent of time that the vehicle is detected, is referred to as the occupancy or occupancy ratio.

E

\section{Effective Cycle Length}

The actual amount of time that is required by a signal controller to serve all of the movements at an intersection. This is in contrast to a programmed value of cycle length. A difference can sometimes occur when actuation is used. Also, the cycle might be interrupted by preemption or transit priority. Furthermore, under non-coordinated control and with some types of adaptive control, there may not be a programmed cycle length. However, the signal will nonetheless use a certain amount of time to rotate through all of the competing movement at the intersection. This is the effective cycle length. 


\section{Eight-Phase Operation}

The most common type of intersection control used at the intersection of two roadways in North America. Each approach has right-turn and through movements (often grouped together), as well as a left-turn movement. These constitute the eight phases served by the signal cycle. The numbering scheme for eight-phase control often serves as a template for other intersection types.

\section{Equivalent Hourly Capacity}

The theoretical maximum amount of traffic (in vehicles per hour) that could be served on a movement under the actual duration of green time provided by the traffic signal. The name arises because this metric represents the amount of green time as the equivalent amount of traffic that would be moved at capacity.

$\mathbf{F}$

\section{Fixed Cycle}

Refers to the method which most coordinated signal controllers operate. The cycle length is fixed and does not substantially vary from cycle to cycle, typically between 90 seconds to three minutes. Cycle lengths are usually set by time of day to accommodate changes in traffic patterns. Some traffic responsive and adaptive control strategies can adjust the cycle lengths in response to measured traffic conditions.

\section{Floating Car Study}

A method used to sample the travel time on a corridor. The analyst drives a vehicle along the corridor and records the amount of time it takes to traverse the system, as well as the clock time at designated landmarks. The driver is instructed to travel at a speed representative of other traffic. This process yields about one travel time sample every 15 to 30 minutes, depending on the length of the corridor.

\section{Force Off}

A condition where an actuated signal phase terminates so that the controller can return to green on the coordinated phases by the scheduled time. Vehicle detection caused the phase to be extended for as long as possible; if there had been a gap in the detection, the phase would have ended with a gap out instead. The amount of time available to the phase is controlled by the split and the cycle length. A force off indicates that the phase might have needed more green time than was available during that cycle. Force off only occurs during coordinated operations. During non-coordinated operations, the maximum green timer is used instead.

\section{Free Flow Travel Time}

The length of time required to traverse a roadway between two designated landmarks while traveling under "free flow" conditions. Free flow means that drivers are able to travel at their desired speed, and are unaffected by interaction with other vehicles, traffic control, or inclement weather and other incidents. Free flow travel time represents ideal conditions, which seldom occur in reality, but is helpful to give perspective to measured travel times. 
G

\section{Gap Out}

A condition where an actuated signal phase is terminated by the detection of a gap in the traffic flow by vehicle detectors. A gap out indicates that the provided green was adequate to serve the phase, because more green time would have been available if more traffic had been present.

\section{Green Occupancy Ratio (GOR)}

The proportion of the green time in which a stop bar detector is occupied at a particular movement and/or lane at an intersection. A high GOR indicates high utilization. A low GOR reflects low utilization.

\section{H}

\section{High-Resolution Controller Data}

Data logged at a traffic signal controller that consists of timestamped events as they are observed by the controller. The "high-resolution" term serves to contrast this type of data with legacy measures of volume and occupancy that are usually aggregated in bins ranging from 1 to 15 minutes. The high-resolution data captures three basic types of events:

Vehicle detections, which describe when each detector becomes occupied or unoccupied. This measures the behavior of traffic at the intersection or on approach to the intersection.

- Signal output changes (e.g., when a signal head changes to green, yellow, or red). This measures the allocation of capacity and the schedule of green times.

- Other events internal to the controller that are relevant to traffic control decision making, such as pattern changes or preemption inputs.

\section{Highway / Freeway}

A roadway that emphasizes throughput over land-access, and is characterized by gradeseparated interchanges.

I

\section{Incident}

An unplanned, non-recurring, and generally unpredictable exceptional circumstance that negatively affects traffic performance. While the definition of "incident" varies by agency, it typically includes traffic accidents, cargo spillages, and disabled vehicles. Some may also consider road work and special events that generate heavy traffic demands as incidents. Incidents generally induce a heavy amount of delay. 


\section{Infrastructure}

Within the context of transportation, this term refers to physical assets such as roadways, interchanges, and bridges.

Infrastructure Quality

Metrics that reflect the state of repair, functionality, and ability of infrastructure to meet its designed purpose.

\section{Interquartile Range (IQR)}

The difference between the $75^{\text {th }}$ and $25^{\text {th }}$ percentiles.

\section{Intersection}

An at-grade crossing of two roadways. The key characteristic of an intersection is that the main through-lanes of the two intersections share the same pavement, and at least one roadway must stop or yield to the other. Intersections usually feature traffic control devices such as traffic signals. This contrasts with grade-separated crossings where the main through-lanes are separated by a bridge, and turns are made using ramps.

\section{Intersection Capacity Utilization}

Refers to the percent of capacity utilized at an intersection. If demand exceeds capacity, oversaturated conditions exist. If demand is less than capacity by a large percentage, then under-saturation exists, also termed under-utilization. Intersection capacity utilization describes the overall demand for capacity at an intersection and is independent of the actual allocation of the capacity. Individual movements and phases can be assessed with the volume-to-capacity ratio, or by metrics based on detector occupancy, such as the Green Occupancy Ratio and Red Occupancy Ratio.

\section{Intersection Control Plans}

The parameters governing local control at an intersection, such as the duration of yellow times or the duration of passage times for detector actuation. The control plan may vary by time of day and may include coordination parameters.

$\mathbf{K}$

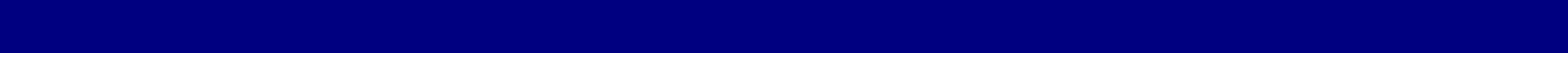

$\mathbf{L}$

\section{Land Access}

The ability of a roadway to permit users to reach facilities on abutting lands, such as driveways. Roadways are divided into different functional classes by considering the degree to which they permit land access, as opposed to throughput. A freeway system provides no land access, while local roads provide full land access. Arterials typically fall somewhere in the middle. 


\section{Local Street / Road}

Roadways whose primary purpose is to provide land access to either homes or businesses and services. In contrast to arterials which balance throughput with land access, or freeways, whose sole purpose is throughput.

\section{M}

\section{Max Out}

A condition where an actuated signal phase terminates because its maximum green timer has expired. Vehicle detection caused the phase to be extended for as long as possible; if there had been a gap in the detection, the phase would have ended with a gap out instead. The amount of time available to the phase is controlled by the "maximum green" setting. A max out indicates that the phase might have needed more green time than was available during that cycle. Max out usually only occurs during non-coordinated operations. During coordinated operations, phases are ended with force off instead.

\section{Mobility}

Within the context of roadway transportation, the ability of a transportation system to move individuals and goods from one location to another.

\section{Movements (Turning Movements)}

The various paths that vehicles need to traverse through an intersection. Movements typically include right, through, and left from each approach.

\section{Moving Ahead for Progress in the 21st Century Act (MAP-21)}

Act signed into law by President Obama on July 6, 2012 funding surface transportation programs. MAP-21 is the first long-term highway authorization enacted since 2005. It emphasizes the application of performance measures and management.

\section{Metropolitan Planning Organization (MPO)}

A cooperative organization within a metropolitan area charged with developing and maintaining long term roadway improvement programs and plans.

\section{$\mathbf{N}$}

\section{Network}

The system of roadways that deliver mobility to an area or region.

0

\section{Offset}

In traffic signal timing, this parameter controls coordination between adjoining signals. The offset defines the time difference between a local cycle reference point (such as the end of 
green for the arterial through movement) and a system cycle reference point. This allows the schedule of green signals at neighboring intersections to be arranged in such a way to allow traffic departing one intersection to progress through other intersections in the system without stopping.

\section{Origin-Destination}

Origin-Destination (or simply OD) is a general term reflecting the overall pattern of the locations where trips begin and end. Each individual trip has an origin and a destination, such as a trip from "home" to "work" or vice versa. The OD patterns in an area comprise many such individual trips. The OD characteristics of a region do not necessarily reflect the roadways or paths upon which the trips are taken. Often, there are many alternative routes between an origin and a destination.

$\mathbf{P}$

\section{Pareto Sorted List}

A list sorted in descending order of frequency.

\section{Percent on Green (POG)}

The percentage of arrivals at a signalized intersection that arrive during the green phase, and are allowed to progress through without stopping.

\section{Performance Measures / Performance Measurement}

In the context of this report, a tool that enables the assessment and comparison of a roadway facilities. Performance measures may consist of quantitative values or, in some cases, visualizations of quantitative data. Ideally, performance measures are based on objective measurements of traffic, and reflect progress toward an objective.

\section{Platoon}

Vehicles grouped together while traversing along a corridor. Platoons of vehicles may be formed through operation of signal systems when signals are coordinated to maximize the probability of progressing through intersections without stopping.

\section{Progression}

This term refers to the ability of a traffic signal system to move platoons along corridors efficiently; i.e. traffic is progressed through successive intersections without stopping. This is a vital function of an arterial roadway.

\section{Purdue Coordination Diagram (PCD)}

A standard chart constructed from high-resolution controller data which provides an effective visualization reflecting the quality of progression for a particular movement through an intersection.

Q

\section{Queue, Queuing}

A queue is a line of stopped vehicles. In the context of arterial traffic management, queues 
form as arriving vehicles stop at red signals, and they disperse as vehicles are released during green. The cyclic pattern of vehicle arrivals, queuing, and departures is characteristic of traffic signal control. Signal coordination strategies can reduce queuing by scheduling vehicle arrivals to coincide with green. However, queuing can almost never be eliminated completely, because of variations in driver speed, traffic entering from turning movements and driveways, and other factors.

$\mathbf{R}$

\section{Red Occupancy Ratio (ROR)}

The proportion of the first five seconds of red in which a stop bar detector is occupied at a particular movement and/or lane at an intersection. A high ROR indicates that vehicles were present at the end of green. A low ROR indicates that vehicles were not present. When a high ROR is combined with a high GOR for the preceding green interval, it is likely that a split failure occurred.

\section{Reliability}

The ability of a roadway, and its supporting systems to provide a predictable level of mobility to its users. Although a general concept, reliability is frequently measured as a statistical travel time parameter, for example, the 95th percentile travel time. However, standard measures have not been established for arterial roadways.

\section{S}

\section{Safety}

Within the context of transportation, attributes of roadways or intersections whose primary purpose is the safety of the traveling public. Examples include width of shoulder and red clearance times. Also encompasses programs that encourage seat belt usage.

\section{Signal Control Data Logger}

A device that records high-resolution controller data. The data is then transferred to a database for analysis.

\section{Signal State Change}

A change in the state of any display at a traffic signal, such as from red to green, green to yellow, or yellow to red, "don’t walk” to "walk," etc.

\section{Spillback}

The condition when queues extend far enough to affect the adjoining upstream intersection. This occurs most often during oversaturation, although closely spaced intersections may Split experience spillback under moderate traffic conditions.

The amount of time within a cycle dedicated to service of a particular phase or movement.

\section{Split Failure}

In the context of the report, this refers to the condition where not all of the demand for service on a movement is able to be served during an instance of green. That is, there was 
queued traffic waiting for service at the beginning of green, and some of this traffic remained at the end of green. That traffic must wait through the following red interval, until the next beginning of green, before it can be served. Because each unserved vehicle experiences an increase in their delay close to a cycle length, a split failure is an indicator of poor service. While an occasional split failure is tolerable, recurring split failures, especially those that occur for several cycles in succession, indicate that there is a systematic problem where the movement is becoming oversaturated. A growing queue of vehicles might lead to spillback of queues, or interference with queues in other lanes. When split failures occur on multiple phases, this is called a cycle failure.

T

\section{Throughput}

The ability of a roadway to move traffic along its length. Roadways are divided into different functional classes by considering the degree to which they emphasize throughput, as opposed to land access. A freeway system provides a high level of throughput (but no land access), while local roads provide less throughput and heavy land access. Arterials typically fall somewhere in the middle.

\section{Time-Space Diagram}

A graphical method to observe the quality of progression through a series of signalized intersections in two directions along a roadway. One axis represents "time" and the other axis represents "distance.” The signal state is represented by bars that indicate what times the signal is red or green. The quality of progression can be shown either by lines that show vehicle trajectories, or by "bands" that indicate where progression between intersections would be provided by the scheduled green times.

\section{Timestamp}

The date and time of an event or data record.

\section{Timing Plans, System Timing Plans}

The timing plans that are used at an intersection, along with the time of day in which they are put into effect, and any coordination parameters used with adjoining intersections.

\section{Traffic Control}

Methods used to direct traffic, ensuring safe mobility. Traffic signals are one example. Other examples include stop signs, and roundabouts.

\section{Travel Time}

The length of time required to traverse a roadway between two designated landmarks.

\section{Turning Movement Counts}

The measured number of vehicles that approach an intersection and the direction in which they proceed. Traditional turning movement counts are performed manually by observing traffic and recording the data. 


\section{U}

\section{User-Oriented Objectives}

This phrase is used in contrast to 'agency-oriented objectives', and reflects the issues and goals critical to a user of the system. Such objectives are typically aligned with the experience of the driver. Such objectives typically include:

- Maintain smooth traffic flow by scheduling and sequencing service (green) times at neighboring signalized intersections

- Minimize the waiting times of stopped traffic at side streets

- Distribute capacity as fairly and efficiently as possible across movements and modes

- Provide adequate service times for non-motorized modes at signalized intersections

- Provide the ability for transit vehicles to execute loading and unloading activities and prioritize transit vehicle service to expedite travel times

- Minimize the amounts of pollution and noise by improving the efficiency of vehicle movement through the system.

V

\section{Vehicle Re-Identification Data}

Measurements of the amount of time it takes a vehicle to travel between two distinct points in a roadway network. This is accomplished by identifying a vehicle at two locations using a sensor and logging the location and exact time of each observation.

\section{Volume}

The number of vehicles that traverse a roadway, or through an intersection, in a given amount of time. Typically measured in vehicles per hour.

\section{Volume-to-Capacity ratio (V/C)}

A measure of the degree to which the capacity provided to a movement at a signalized intersection is utilized by traffic. The $\mathrm{v} / \mathrm{c}$ is obtained by dividing the measured traffic volume by the capacity (determined from the duration of green). A high $\mathrm{v} / \mathrm{c}$ ratio indicates a movement that is likely to experience split failures.

\section{W}




\section{Report Sponsor}

The "Small Business Innovation Development Act of 1982" (Pub. L. No. 97-219), along with reauthorizing legislation (Pub. L. No. 99-443 and Pub. L. No. 102-564, the "Small Business Research and Development Enhancement Act of 1992"), seeks to encourage the initiative of the private sector and to use small business effectively to meet federal research and development objectives. To comply with statutory obligations of the Act, the U.S. Department of Transportation established the Small Business Innovation Research (SBIR) Program, which conforms to the guidelines and regulations provided by the Small Business Administration. Annually, small businesses are solicited to submit innovative research proposals that address the high-priority requirements of the U.S. Department of Transportation and that have potential for commercialization.

This report was developed through a partnership between Traffax, Inc., and Purdue University with funding from a Phase III SBIR contract (DTFH6114C00035) with the Federal Highway Administration. The project, entitled "Sensor Fusion and MOE Development for Off-Line Traffic Analysis of Real Time Data," created and refined methods and tools for the characterization of performance along arterial corridors.

\section{Publication}

This report is part of a series of reports published in collaboration with USDOT, Traffax, Inc., and Purdue University. The full report series is available for download at http://docs.lib.purdue .edu/apmtp/.

\section{Open Access and Collaboration with Purdue University}

The Indiana legislature established the Joint Highway Research Project in 1937. In 1997, this collaborative venture between the Indiana Department of Transportation and Purdue University was renamed as the Joint Transportation Research Program (JTRP) to reflect state and national efforts to integrate the management and operation of various transportation modes. Since 1937, the JTRP program has published over 1,600 technical reports. In 2010, the JTRP partnered with the Purdue University Libraries to incorporate these technical reports in the University's open access digital repository and to develop production processes for rapidly disseminating new research reports via this repository. Affiliated publications have also recently been added to the collection. As of 2017, the JTRP collection had over 1.5 million downloads, with some particularly popular reports having over 20,000 downloads. 